\title{
Traumatismes de L'abdomen en Milieu Africain : Aspects Épidémiologiques, Diagnostiques, et Thérapeutiques
}

\author{
Belemlilga G. L. Hermann, \\ Zaré Cyprien,
}

Institut Supérieur des Sciences de la Santé, Université Nazi Boni, Centre

Hospitalier Universitaire Sourô Sanou, Bobo-Dioulasso, Burkina Faso

Yabré Nassirou,

Keita Namori,

Benao B. Lazare,

Département de Chirurgie et Spécialités Chirurgicales, Centre Hospitalier

Universitaire Sourô Sanou, Bobo-Dioulasso, Burkina Faso

\section{Somé O. Roland,}

Sanon B. Gustave,

Institut Supérieur des Sciences de la Santé, Université Nazi Boni, Centre Hospitalier Universitaire Sourô Sanou, Bobo-Dioulasso, Burkina Faso

Doi:10.19044/esj.2020.v16n21p132 URL:http://dx.doi.org/10.19044/esj.2020.v16n21p132

\section{Résumé}

Introduction : La prise en charge des plaies et contusions de l'abdomen est bien codifiée avec l'existence d'algorithmes. Au Burkina Faso, les ressources humaines, les moyens diagnostiques et thérapeutiques sont limités. A cet égard, l'objectif de cette étude était d'étudier les aspects épidémiologiques, diagnostiques et thérapeutiques des traumatismes de l'abdomen. Patients et méthodes : L'étude en question était descriptive rétrospective, durant deux ans allant de janvier 2017 à décembre 2018 au CHU Sourô Sanou de Bobo-Dioulasso. Les patients inclus avaient un diagnostic de plaie ou de contusion de l'abdomen. Les patients ayant un dossier incomplet ont été exclus. Résultats : Durant l'étude l'information de 63 patients a été colligée, 51 hommes et 12 femmes. L'âge moyen était de 22 ans. Les accidents de la circulation routière étaient au nombre de 25 . Parmi ces traumatismes figuraient 39 contusions et 24 plaies de l'abdomen. Une instabilité hémodynamique était présente chez 15 patients. Les présentations cliniques étaient : 25 cas de douleurs abdominales, 14 cas d'hémopéritoine, 12 cas de syndrome péritonéal, 9 cas d'éviscération. Les lésions de la rate étaient 
présentes dans huit cas. Les polytraumatismes étaient au nombre de 16. L'échographie abdominale était réalisée dans 19 cas, l'ASP dans 22 cas. Le traitement chirurgical a été réalisé chez 26 patients. La laparotomie était non thérapeutique (ne nécessitant pas de geste chirurgical spécifique) chez 15 patients. Les complications postopératoires comportaient cinq cas de suppuration pariétale, un cas de péritonite postopératoire. Le décès était survenu chez huit (12,7\%) patients et la durée d'hospitalisation moyenne était de 8,5 jours. Conclusion : Les traumatismes de l'abdomen concernent les adultes jeunes de sexe masculin, et sont dus aux accidents de la circulation routière. L'ASP et l'échographie au lit du patient ne sont pas réalisées. Le taux de laparotomie non thérapeutique est élevé ainsi que la mortalité.

Mots-clés : Traumatisme Abdominal, Épidémiologie, Diagnostic, Traitement, Burkina Faso

\title{
Trauma to the Abdomen in African Environment: Epidemiological, Diagnostic, and Therapeutic Aspects
}

\section{Belemlilga G. L. Hermann, Zare Cyprien,}

Institut Supérieur des Sciences de la Santé, Université Nazi Boni, Centre Hospitalier Universitaire Sourô Sanou, Bobo-Dioulasso, Burkina Faso

\section{Yabré Nassirou, Keita Namori, Benao B. Lazare,}

Département de Chirurgie et Spécialités Chirurgicales, Centre Hospitalier Universitaire Sourô Sanou, Bobo-Dioulasso, Burkina Faso

\section{Some O. Roland,}

Sanon B. Gustave,

Institut Supérieur des Sciences de la Santé, Université Nazi Boni, Centre Hospitalier Universitaire Sourô Sanou, Bobo-Dioulasso, Burkina Faso

\begin{abstract}
Introduction: The management of wounds and contusions of the abdomen is well codified with the existence of algorithms. In Burkina Faso, human resources, diagnostic and therapeutic means are limited. This paper focuses on studying the epidemiological, diagnostic, and therapeutic aspects
\end{abstract}


of trauma in the abdomen. Patients and Methods: This study employs a descriptive retrospective for two years beginning from January 2017 to December 2018 in Sourô Sanou University Hospital of Bobo-Dioulasso. The patients included in the study were diagnosed with a wound or contusion of the abdomen. Patients with incomplete records were excluded. Results: During the study, the data of 63 patients were collected, 51 men and 12 women. The mean age was 22 years old. There were 25 road traffic accidents. Among these injuries were 39 bruises and 24 abdominal wounds. Hemodynamic instability was present in 15 patients. The clinical presentations were: 25 cases of abdominal pain, 14 cases of hemoperitoneum, 12 cases of peritoneal syndrome, and 9 cases of evisceration. Lesions of the spleen were present in 8 cases and there were 16 polytrauma cases. Abdominal ultrasound was performed in 19 cases, abdominal X-rays in 22 cases. Surgical treatment was carried out in 26 patients. Laparotomy was non-therapeutic (not requiring a specific surgical procedure) in 15 patients. Postoperative complications included five cases of parietal suppuration, one case of postoperative peritonitis. Death occurred in eight $(12.7 \%)$ patients. The average length of hospital stay was 8.5 days. Conclusion: Trauma to the abdomen is of interest to young adult males, and it occurs majorly due to traffic accidents. Abdominal $\mathrm{x}$-rays and patient bedside ultrasound are not performed. The rate of nontherapeutic laparotomy is high as well as the mortality.

Keywords: Abdominal Trauma, Epidemiology, Diagnostic, Treatment, Burkina Faso

\section{Introduction}

A l'échelle mondiale, les blessures par violences ou accidents occupent le deuxième rang des causes de mortalité (Hoffmann et al., 2013). Ainsi, dans les pays peu développés d'Afrique, un problème de santé publique tel que les traumatismes (Odimba, 2007) est aggravé par le manque de ressources. Les traumatismes de l'abdomen sont mortels par leur risque hémorragique et septique. La prise en charge des plaies et contusions de l'abdomen est bien codifiée avec l'existence d'algorithmes. Elle est pluridisciplinaire, nécessitant des moyens importants et parfois des centres spécialisés (Mutter et al., 2005). Pour cela on utilise des moyens tels que : réanimation pré-hospitalière, FAST (Focus Assessment with Sonography for Trauma), écho et radiographie au lit du patient, radiologie interventionnelle, laparoscopie, damage control, trauma center, qui permettent désormais une chirurgie sélective.

$\mathrm{Au}$ Burkina Faso, les ressources humaines, les moyens diagnostiques et thérapeutiques sont limités. Dans ce sens, le but de ce travail était d'étudier les aspects épidémiologiques, diagnostiques et thérapeutiques des 
traumatismes de l'abdomen au Centre Hospitalier Universitaire Sourô Sanou (CHUSS) de Bobo-Dioulasso.

\section{Patients et méthodes}

Le cadre de cette étude était le service des urgences chirurgicales du CHUSS de Bobo-Dioulasso où sont reçues les urgences chirurgicales : générales et viscérales, urologiques, traumatologiques et orthopédiques, ORL, maxillo-faciales, odonto-stomatologiques, ophtalmologiques. Ce service polyvalent comportait une salle d'accueil, deux salles de mise en observation, une salle de déchoquage, deux salles opératoires. Le service de réanimation polyvalente, le service d'imagerie médicale et le laboratoire d'analyses médicales étaient communs à tous les services de l'hôpital et n'étaient pas situés au sein des urgences chirurgicales.

Ce travail est une étude descriptive rétrospective allant du 01 janvier 2017 au 31 décembre 2018, soit deux ans. Tous les dossiers des patients admis aux urgences chirurgicales du CHUSS dans la période d'étude ont été inclus, chez qui le diagnostic de plaie ou de contusion de l'abdomen avait été retenu. Le diagnostic de plaie de l'abdomen était évoqué cliniquement devant une rupture de la continuité de la paroi abdominale suite à un traumatisme. Le diagnostic de contusion était évoqué cliniquement devant un traumatisme de l'abdomen sans rupture de la continuité pariétale. L'abdomen était défini comme la région située entre le diaphragme et le plancher pelvien. Les dossiers avec des données incomplètes ont été exclus. Les variables étudiées étaient : l'âge, le sexe, le délai de consultation, les circonstances du traumatisme, l'agent vulnérant, les paramètres cliniques, les examens paracliniques, la nature du traitement, les gestes chirurgicaux, les complications, la mortalité, la durée d'hospitalisation.

\section{Résultats}

63 cas de traumatisme de l'abdomen ont été colligés, soit 1,3\% des admissions en urgence en chirurgie générale et viscérale. Il s'agissait de 51 $(80,9 \%)$ hommes et $12(19,1 \%)$ femmes. Le sex-ratio était de 4,2. L'âge moyen était de 22 ans avec des extrêmes de un et 90 ans. Les accidents de la circulation routière étaient la principale circonstance de ces traumatismes avec $25(39,7 \%)$ cas. Parmi les traumatismes, il y avait 39 (61,9\%) contusions, 24 $(38,1 \%)$ plaies de l'abdomen. Le Tableau I montre la répartition des patients selon le type de traumatisme et les circonstances de survenue. L'agent vulnérant dans les plaies de l'abdomen était : arme blanche (neuf cas), balle (un cas), bris de verre (deux cas), bois (un cas), corne de bovidé (six cas), inconnu (six cas). Le délai de consultation était supérieur à 24 heures dans 14 $(22,2 \%)$ cas. Une instabilité hémodynamique a été notée chez $15(23,8 \%)$ patients. Les présentations cliniques étaient les suivantes : $25(39,7 \%)$ cas de 
douleurs abdominales, $14(22,2 \%)$ cas d'hémopéritoine, 12 cas (19\%) de syndrome péritonéal, neuf $(14,3 \%)$ cas d'éviscération, deux cas d'agent vulnérant in situ, un cas d'hématurie. La répartition des patients selon le type de plaie et le siège de l'orifice d'entrée des plaies est présentée au Tableau II. Parmi les traumatismes, il y avait $20(31,7 \%)$ contusions avec lésion viscérale, $20(31,7 \%)$ plaies pénétrantes et $13(20,6 \%)$ plaies perforantes. Il n'y avait pas de plaie transfixiante. Les plaies pénétrantes étaient confirmées neuf fois cliniquement devant une éviscération, six fois après une exploration sous anesthésie locale et cinq fois après exploration chirurgicale sous anesthésie générale. Les lésions de la rate étaient présentes dans huit cas $(12,7 \%)$, et celles du foie dans six cas $(9,5 \%)$. La répartition des patients selon le type de traumatisme et les organes lésés est présentée au Tableau III. Les polytraumatismes étaient au nombre de $16(25,4 \%)$. Les traumatismes associés étaient : 10 cas $(15,9 \%)$ de traumatisme de membres, 4 cas $(6,3 \%)$ de traumatisme cranio-encéphalique, 4 cas $(6,3 \%)$ de traumatisme thoracique. L'échographie abdominale était réalisée dans $19(30,1 \%)$ cas ; la radiographie de l'abdomen sans préparation dans $22(34,9 \%)$ cas avait montré un pneumopéritoine dans un cas. Le traitement chirurgical a été réalisé chez 26 $(41,3 \%)$ patients. Les gestes chirurgicaux réalisés en fonction des organes lésés figurent dans le Tableau IV. La laparotomie était non thérapeutique (ne nécessitant pas de geste chirurgical spécifique) chez 15 (57,7\%) patients dont 11 cas de plaie pénétrante. $10(38,5 \%)$ cas de laparotomie blanche ont été notés dont neuf plaies pénétrantes. Les complications postopératoires étaient : cinq cas de suppuration pariétale, un cas de péritonite postopératoire, un cas de choc hypovolémique. Le décès est survenu chez huit (12,7\%) patients dont six par contusion et deux par plaie pénétrante abdominale. Parmi les décès deux polytraumatismes ont été notés (hémothorax et fracture bilatérale du fémur), six décès avant 24 heures et deux décès entre 24 et 48 heures. Il n'y a pas eu de décès post-opératoire. La durée d'hospitalisation moyenne était de 8,5 jours avec des extrêmes d'un et 43 jours.

Tableau I. Répartition des patients selon le type de traumatisme et les circonstances de survenue $(n=63)$

\begin{tabular}{|c|c|c|c|c|c|c|c|}
\hline Traumatisme & ACR $^{\mathrm{a}}$ & $\begin{array}{c}\text { Encorne- } \\
\text { ment }\end{array}$ & $\begin{array}{c}\text { Chute de } \\
\text { hauteur }\end{array}$ & $\begin{array}{c}\text { Arme } \\
\text { blanche }\end{array}$ & $\begin{array}{c}\text { Arme } \\
\text { à feu }\end{array}$ & Autres $^{\mathrm{b}}$ & Total \\
\hline Contusion & 20 & 4 & 9 & 0 & 0 & 6 & 39 \\
\hline Plaie & 5 & 7 & 1 & 9 & 1 & 1 & 24 \\
\hline Total & 25 & 11 & 10 & 9 & 1 & 7 & 63 \\
\hline
\end{tabular}

${ }^{\mathrm{a}}$ Accident de la Circulation Routière. ${ }^{\mathrm{b}}$ Accident ludique, coup de sabot, sport, éboulement, autolyse. 
Tableau II. Répartition des patients selon le type de plaie et le siège de l'orifice d'entrée $(n=24)$

\begin{tabular}{|c|c|c|c|}
\hline Siège de l'orifice d'entrée & Plaie pénétrante & Plaie non pénétrante & Total \\
\hline Région latérale gauche & 7 & 1 & 8 \\
\hline Région latérale droite & 4 & 0 & 4 \\
\hline Région ombilicale & 4 & 0 & 4 \\
\hline Epigastre & 1 & 1 & 2 \\
\hline Hypochondre & 0 & 1 & 1 \\
\hline Région inguinale droite & 0 & 1 & 1 \\
\hline Région inguinale gauche & 0 & 1 & 1 \\
\hline Région lombaire droite & 1 & 0 & 1 \\
\hline Périnée & 2 & 0 & 2 \\
\hline Total & 19 & 5 & 24 \\
\hline
\end{tabular}

Tableau III. Répartition des patients selon le type de traumatisme et les organes lésés $(\mathrm{n}=27)$

\begin{tabular}{|c|c|c|c|c|c|c|c|c|}
\hline $\begin{array}{c}\text { Traumatism } \\
\mathbf{e}\end{array}$ & $\begin{array}{c}\text { Foi } \\
\mathbf{e}\end{array}$ & $\begin{array}{c}\text { Rat } \\
\mathbf{e}\end{array}$ & $\begin{array}{c}\text { Mésentèr } \\
\mathbf{e}\end{array}$ & $\begin{array}{c}\text { Pancréa } \\
\mathbf{s}\end{array}$ & $\begin{array}{c}\text { Rei } \\
\mathbf{n}\end{array}$ & $\begin{array}{c}\text { Vessi } \\
\mathbf{e}\end{array}$ & $\begin{array}{c}\text { Intesti } \\
\mathbf{n}\end{array}$ & $\begin{array}{c}\text { Tota } \\
\mathbf{l}\end{array}$ \\
\hline Contusion & 6 & 6 & 1 & 1 & 1 & 1 & 2 & 18 \\
\hline Plaie & 0 & 2 & 1 & 0 & 1 & 0 & 5 & 9 \\
\hline Total & 6 & 8 & 2 & 1 & 2 & 1 & 7 & 27 \\
\hline
\end{tabular}

Tableau IV. Répartition des patients opérés selon les organes lésés et les gestes chirurgicaux $(n=16)$

\begin{tabular}{|c|c|}
\hline Geste chirurgical & Nombre \\
\hline Foie & 1 \\
\hline Abstention & 1 \\
\hline Packing & 1 \\
\hline Rate & 1 \\
\hline Abstention & 2 \\
\hline Tamponnement & \\
\hline Splénectomie & 1 \\
\hline Mésentère & 1 \\
\hline Abstention & 2 \\
\hline Suture & \\
\hline Rein & 3 \\
\hline Abstention & 1 \\
\hline Grêle & \\
\hline Suture & 1 \\
\hline Résection anastomose & \\
\hline Colon & 2 \\
\hline Suture + stomie & \\
\hline Rectum & 1 \\
\hline Suture + stomie & \\
\hline Vessie & \\
\hline Abstention & \\
\hline & \\
\hline & \\
\hline & \\
\hline & \\
\hline
\end{tabular}




\section{Discussion}

Les traumatismes de l'abdomen étaient relativement peu fréquents, ils représentaient $1,3 \%$ des admissions en urgence dans cette étude. Cette fréquence est proche de celle trouvée par Raherinantenaina et al. (2015) à Madagascar qui était de $0,2 \%$. En réalité il existe une sous-notification des traumatismes de l'abdomen dans la présente étude. En effet l'étude en question est rétrospective, monocentrique, n'inclut pas les décès préhospitaliers, ni les cas qui auraient pu être diagnostiqués si l'autopsie était réalisée systématiquement.

Les ACR constituaient la première cause des traumatismes de l'abdomen. L'âge moyen était de 22 ans et le sexe masculin était majoritaire. Des publications en Afrique et en Europe ont montré que l'adulte jeune de sexe masculin était la première victime des traumatismes de l'abdomen (Mutter et al., 2005 ; Gaudeuille et al., 2007) qui étaient majoritairement causés par les ACR (Mutter et al., 2005 ; Raherinantenaina et al., 2015). Cela s'explique par le fait qu'au Burkina Faso, en milieu urbain, le transport individuel par véhicule à deux roues est majoritaire, alors que le transport en commun est quasi inexistant. Ces véhicules à deux roues sont couramment utilisés par les jeunes, notamment les élèves et les étudiants.

Cependant, les étiologies des traumatismes abdominaux varient selon le contexte : guerre, violences civiles (Mutter et al., 2005 ; Raherinantenaina et al., 2015). Ainsi, les étiologies observées étaient : en Europe les accidents de la voie publique, aux États-Unis les agressions par arme à feu et arme blanche, en zone de guerre les armes à feu (Mutter et al., 2005). Les lésions dépendent de la nature de l'agent vulnérant, les armes à feu et les armes blanches déterminant des lésions à type de plaie (Gaudeuille et al., 2007), et les ACR des contusions (Gaudeuille et al., 2007 ; Choua et al., 2017 ; Pande et al., 2017).

Cette étude montre une prédominance des contusions de l'abdomen $(61,9 \%)$ sur les plaies de l'abdomen $(38,1 \%)$. Les plaies pénétrantes représentaient $31,7 \%$ des traumatismes. Raherinantenaina et al. (2015) rapportaient $52,6 \%$ de contusion et $47,4 \%$ de plaies de l'abdomen. Par contre Gaudeuille et al. (2007) en Centre Afrique observaient $75 \%$ de plaies et $25 \%$ de contusions. De même, la fréquence des lésions est liée à leurs étiologies. En effet dans le contexte de la présente étude, les ACR, principales causes des contusions, étaient beaucoup plus fréquents que les rixes et les agressions de bovidé, principales causes des plaies de l'abdomen. D'ailleurs, les armes à feu et les rixes (armes blanches) (Gaudeuille et al., 2007) étaient responsables des plaies de l'abdomen. Mais quelle que soit la lésion, les risques des traumatismes de l'abdomen sont hémorragiques et septiques. Le traitement initial consiste en l'hémostase et à la lutte contre l'infection. 
Les lésions de la rate étaient présentes dans 8 cas (12,7\%), et celles du foie dans 6 cas $(9,5 \%)$, celles de l'intestin (grêle, colon, rectum) dans 7 $(11,1 \%)$ cas. Les lésions intestinales étaient surtout présentes dans les traumatismes ouverts, ce qui correspond aux résultats trouvés par Raherinantenaina et al. (2015), et Gaudeuille et al. (2007). Le tube digestif est le plus souvent lésé dans les plaies pénétrantes (Raherinantenaina et al., 2015 ; Gaudeuille et al., 2007 ; Menegaux, 2004), et la réparation de ses lésions est chirurgicale. Par contre, les lésions du foie et de la rate peuvent avoir un traitement conservateur, non chirurgical, faisant appel à la simple surveillance ou à la radiologie interventionnelle.

Les présentations cliniques étaient variables : $14(22,2 \%)$ cas d'hémopéritoine, 12 cas $(19 \%)$ de syndrome péritonéal, neuf $(14,3 \%)$ cas d'éviscération, $15(23,8 \%)$ cas avec une instabilité hémodynamique. Classiquement les traumatisés de l'abdomen sont divisés en deux groupes selon l'état hémodynamique (Menegaux, 2004). On distingue premièrement les patients stables sur le plan hémodynamique, et chez qui on peut réaliser des explorations paracliniques. Deuxièmement les patients instables, chez qui l'exploration chirurgicale est nécessaire si l'instabilité persiste. Les patients présentant une péritonite ou une éviscération sont également traités par laparotomie. Mais l'examen clinique si fondamental dans les traumatismes abdominaux n'est pas toujours contributif(Menegaux, 2004), d'où la nécessité de réaliser des examens paracliniques pour une décision thérapeutique adéquate. Dans ce sens, l'échographie abdominale était réalisée dans 19 $(30,1 \%)$ cas, l'ASP dans $22(34,9 \%)$ cas. La contribution des examens paracliniques dans la prise en charge était peu importante. L'examen clinique restait donc capital du fait de l'indisponibilité des autres moyens diagnostiques.

Le traitement chirurgical par laparotomie était réalisé chez 26 (41,3\%) patients dont 20 cas de plaie pénétrante. La laparotomie était non thérapeutique chez 15 patients. Selon Raherinantenaina et al. (2015) 91,8\% des patients étaient opérés et la laparotomie était blanche ou non thérapeutique dans 15\%. Également, Gaudeuille (2007) notait 16,6\% de laparotomie blanche. Dans cette étude tous les cas de plaie pénétrante ont été explorés chirurgicalement par laparotomie. Cette conduite n'est plus systématique car $20 \%$ des laparotomies systématiques pour plaie pénétrante sont non thérapeutiques (Menegaux, 2004). Le taux élevé de laparotomie non thérapeutique s'explique par l'indisponibilité d'examens paracliniques à visée diagnostique et thérapeutique malgré la nécessité de prendre une décision thérapeutique en urgence. Elle s'explique également par l'indisponibilité de la laparoscopie en urgence. Le recours systématique à la laparotomie dans les plaies pénétrantes se justifie par les difficultés de mise en œuvre d'une surveillance armée qui doit respecter des critères précis dont l'expérience de 
l'équipe médicochirurgicale et la disponibilité d'un plateau technique performant sont les éléments majeurs (Menegaux, 2004).

Le décès était survenu chez $8(12,7 \%)$ patients dont deux polytraumatismes. Il n'y avait pas de décès post-opératoire. Le taux de mortalité était élevé et supérieur à celui de Raherinantenaina et al. (2015) $(5,7 \%)$. Le pronostic vital des blessés est lié en urgence au risque hémorragique et à distance à la péritonite (Menegaux, 2004). Dans le contexte de cette étude les capacités de réanimation et d'hémostase chirurgicale en urgence sont limitées.

\section{Conclusion}

Les traumatismes de l'abdomen sont peu fréquents, avec pour cible principale les adultes jeunes de sexe masculin. Les ACR sont l'étiologie principale déterminant surtout des lésions à type de contusion abdominale. Le tableau clinique est souvent peu contributif et les examens paracliniques sont indisponibles en urgence. Le taux de laparotomie non thérapeutique est élevé ainsi que la mortalité. La sensibilisation des populations sur la sécurité routière, l'équipement des services d'urgence et la formation du personnel peuvent contribuer à baisser cette mortalité.

\section{References:}

1. Choua, O., Rimtebaye, K., Yamingue, N., Moussa, K., \& Kaboro, M. (2017). Aspects des traumatismes fermés de l'abdomen opérés à l'Hôpital Général de Référence Nationale de N'Djaména (HGRN), Tchad : à propos de 49 cas. Pan African Medical Journal, 26,50. doi: 10.11604/pamj.2017.26.50.8327

2. Gaudeuille, A., Doui, AD., Ndémanga, JK., Sacko, E., \& Nali, NM. (2007). Abdominal trauma in Bangui (Central Africa). Epidemiologic and anatomical aspects. Mali Med, 22(2),19-22.

3. Hoffmann, C., Goudard, Y., Falzone, E., Leclerc, T., Planchet, M., Cazes, N., Pons, F., Lenoir, B., \& Debien, B. (2013). Prise en charge des traumatismes pénétrants de l'abdomen: des spécificités à connaître. Annales Françaises d'Anesthésie et de Réanimation, 32(2), 104-111. https://doi.org/10.1016/j.annfar.2012.12.006

4. Menegaux Menegaux, F. (2004). Plaies et contusions de l'abdomen. EMC - Chirurgie, 1(1), 18-31. doi: 10.1016/S1762-570X(03)00004-5.

5. Mutter, D., Schmidt-Mutter, C., \& Marescaux, J. (2005). Contusions et plaies de l'abdomen. EMC - Médecine, 2(4), 424-47. doi: 10.1016/j.emcmed.2005.01.001

6. Odimba, E. B. F. K. (2007). Aspects particuliers des traumatismes dans les pays peu nantis d'Afrique. Un vécu chirurgical de 20 ans. emémoires de l'Académie Nationale de Chirurgie, 6 (2), 44-56. 
7. Pande, R., Saratzis, A., Winter Beatty, J., Doran, C., Kirby, R., \& Harmston, C. (2017). Contemporary characteristics of blunt abdominal trauma in a regional series from the UK. The Annals of The Royal College of Surgeons of England, 99(1), 82-7. doi: 10.1308/rcsann.2016.0223

8. Raherinantenaina, F., Rakotomena, S. D., Rajaonarivony, T., Rabetsiahiny, L. F., Rajaonanahary, T. M. A., Rakototiana, F. A., Hunald, F. A., \& Rakoto, R. H. N. (2015). Blunt and penetrating trauma of the abdomen: retrospective analysis of 175 cases and review of literature. The Pan African medical journal, 20, 129. https://dx.doi.org/10.11604/pamj.2015.20.129.5839 